\title{
NUMERICAL BOUNDARY CONDITIONS FOR SCHEMES WITH CENTERED AND BIASED DIFFERENCES IN SUBSONIC GAS DYNAMICS
}

\author{
ILYA V. ABALAKIN ${ }^{1}$ and LUDWIG W. DORODNICYN ${ }^{2}$ \\ ${ }^{1}$ M.V. Keldysh Institute for Applied Mathematics \\ Miusskaia sq. 4, 125047 Moscow, Russia \\ ilya.abalakin@gmail.com \\ ${ }^{2}$ M.V. Lomonosov Moscow State University, Faculty CMC \\ Vorobievy gory, 119991 Moscow, Russia \\ dorodn@cs.msu.su
}

Key words: Gas Dynamics, Aeroacoustics, Euler Equations, Nonreflecting Boundary Conditions, Finite Differences, Edge Based Reconstruction

\begin{abstract}
For finite-difference schemes of EBR class in multi-dimensional inviscid gas dynamics, the nonreflecting boundary conditions are analyzed and developed. The wave-reflection properties of discrete models differ significantly from each other and from the continuous Euler equations. For certain schemes there exist local boundary conditions which lead to small reflections of waves with arbitrary incidence angle. Numerical examples are shown both linear and nonlinear.
\end{abstract}

\section{INTRODUCTION}

Modeling external problems of subsonic gas dynamics or aeroacoustics and similar fields faces spurious wave reflections from artificial boundaries. The reason is in the fact that for the multi-dimensional wave equation it is impossible to specify any local nonreflecting boundary conditions [1]. Alternative and more expensive techniques are used such as nonlocal boundary conditions and Perfectly Matched Layers (PML), see, e.g., [2].

To demonstrate this feature, consider, reproducing our paper [3], the 2D wave equation

$$
\frac{\partial^{2} p}{\partial t^{2}}-\left(\frac{\partial^{2} p}{\partial x^{2}}+\frac{\partial^{2} p}{\partial y^{2}}\right)=0,0<x<X, 0<y<Y, t>0 .
$$

The right-hand boundary condition

$$
\left.(\partial p / \partial t+\partial p / \partial x)\right|_{x=X}=0
$$

is nonreflecting for the 1D case and causes reflection of oblique waves, for $\theta$ the angle of incidence (Fig. 1), with the uniquely determined reflection coefficient $R$ as

$$
\begin{aligned}
& p(x, y, t ; \omega, \theta)=\exp \{i \omega t-i \omega x \cos \theta-i \omega y \sin \theta\} \\
& +R \exp \{i \omega t+i \omega x \cos \theta-i \omega y \sin \theta\}, \quad R=R(\theta)=-\tan ^{2}(\theta / 2) .
\end{aligned}
$$




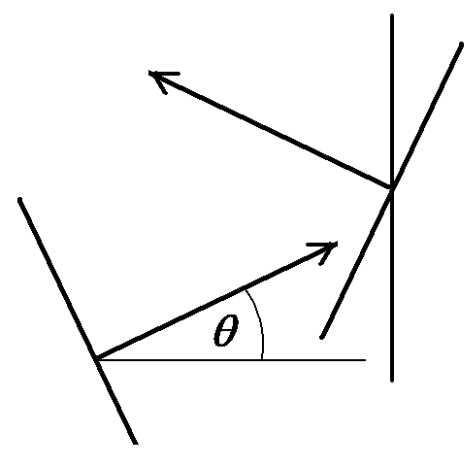

Figure 1: Schematic of wave reflection from the right-hand boundary

However, the situation is not the same when viscous models or discrete equations are used for the fluid description. Finite-difference schemes usually possess physical (regular) as well as additional (singular) modes and require a greater number of equations on boundaries than the continuous models do. Such boundary conditions, known from $[4,5]$, do influence strongly the reflective properties of boundaries. Meanwhile, there are scarce publications where these phenomena were thoroughly examined. For a kind of centered-difference scheme, spurious scheme modes were analyzed in [6] and appropriate boundary conditions were constructed.

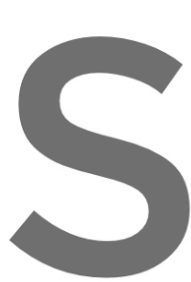

In this study we use numerical sce

and unstructured meshes.

and biased (upwind) diff

For centered-difference sc

tions of oblique waves in the

Snell's law. For upwind sc
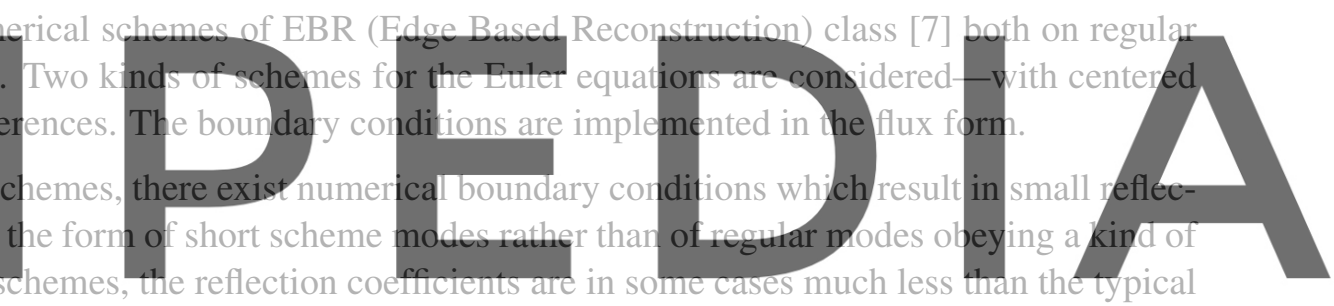

values for the continuous Euler equations.

Register for free at https//www.scipedia.com to download the version without the watermark

2 DIFTERENTIAL EULER EQUATIONS

To make a fundament for further analysis of finite-difference schemes in inviscid gas dynamics, let us start with the simplest case of continuous Euler equations. We mostly repeat our previous paper [3] more briefly.

Consider the linearized 2D Euler equations

$$
\frac{\partial U}{\partial t}+\mathbf{C}_{x} \frac{\partial U}{\partial x}+\mathbf{C}_{y} \frac{\partial U}{\partial y}=0,0<x<X, 0<y<Y, t>0
$$

Here $U=\left(\rho^{\prime} u^{\prime} v^{\prime} p^{\prime}\right)^{T}$ is the vector of perturbations and constant matrices are specified

$$
\mathbf{C}_{x}=\left(\begin{array}{cccc}
u & \rho & 0 & 0 \\
0 & u & 0 & 1 / \rho \\
0 & 0 & u & 0 \\
0 & \rho c^{2} & 0 & u
\end{array}\right), \quad \mathbf{C}_{y}=\left(\begin{array}{cccc}
v & 0 & \rho & 0 \\
0 & v & 0 & 0 \\
0 & 0 & v & 1 / \rho \\
0 & 0 & \rho c^{2} & v
\end{array}\right) .
$$


The general solution to (4) is expanded over normal modes of the four types

$$
U(x, y, t ; \omega, \ell)=\sum_{j=1}^{4} a_{j} \widehat{U}_{j} \exp \left\{i \omega t-i k_{j} x-i \ell y\right\}
$$

The right-going $(j=1)$ and the left-going $(j=2)$ acoustic waves, the entropy $(j=3)$, and the vorticity $(j=4)$ waves are described with wavenumbers $k_{j}$ and eigenvectors

$$
\begin{aligned}
& \left(\begin{array}{llll}
\widehat{U}_{1}(\theta) & \widehat{U}_{2}(\theta) & \widehat{U}_{3}(\theta) & \widehat{U}_{4}(\theta)
\end{array}\right)=\left(\begin{array}{cccc}
\rho & \rho & \rho & 0 \\
c \cos \theta_{1} & c \cos \theta_{2} & 0 & -c \tan \theta_{3} \\
c \sin \theta_{1} & c \sin \theta_{2} & 0 & c \\
\rho c^{2} & \rho c^{2} & 0 & 0
\end{array}\right), \\
& k_{1}=\frac{\omega \cos \theta_{1}}{c+u \cos \theta_{1}+v \sin \theta_{1}}, \quad k_{2}=\frac{\omega \cos \theta_{2}}{c+u \cos \theta_{2}+v \sin \theta_{2}}, k_{3}=k_{4}=\frac{\omega \cos \theta_{3}}{u \cos \theta_{3}+v \sin \theta_{3}} \text {, } \\
& \theta_{1}=\theta, \quad \frac{\sin \theta_{1}}{c+u \cos \theta_{1}+v \sin \theta_{1}}=\frac{\sin \theta_{2}}{c+u \cos \theta_{2}+v \sin \theta_{2}}=\frac{\sin \theta_{3}}{u \cos \theta_{3}+v \sin \theta_{3}} .
\end{aligned}
$$

The full set of relationships is stated, e.g., in [2].

Indicate the case of $1 \mathrm{D}$ Euler equations (angle $\theta=0$ ) for which eigenvectors $\widehat{U}_{j}$, characteristic velocities $\lambda_{j}$, and wavenumbers $k_{j}=\omega / \lambda_{j}$ are the following:
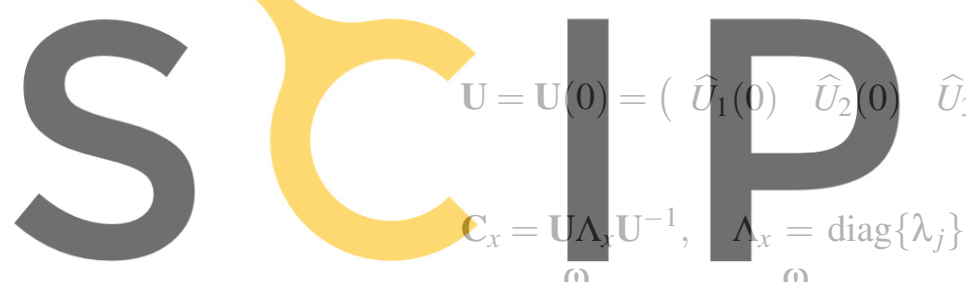

$\omega$

$k_{3}=k_{4}=\frac{\omega}{u}$

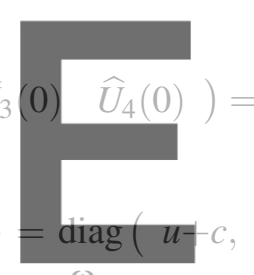

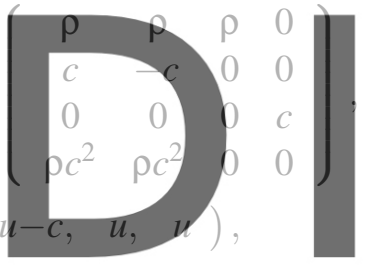

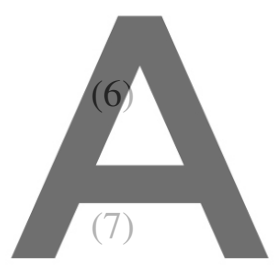

(8)

\section{Register for free at https//www.scipedia.com to download the version without the watermark}

Consider a boundary condition on the right-hand $(x=X)$ or the left-hand $(x=0)$ boundary of the rect-

anguiar domain. If the fiow is subsonic and directed to right,

$$
0<u<c,
$$

the left acoustic wave $\left(k_{2}\right)$ propagates leftward as the rest do rightward. On the left boundary, incoming modes are the right-going acoustic $\left(k_{1}\right)$, the entropy $\left(k_{3}\right)$, and the vorticity $\left(k_{4}\right)$ waves, while outgoing is the left acoustic $\left(k_{2}\right)$. On the right boundary everything is contrary. Consequently, the Euler equations need 3 boundary conditions on the left and 1 condition on the right.

A boundary condition is nonreflecting if it assigns zero amplitudes $a_{j}=0$ to incoming waves and arbitrary $a_{j}$ to outgoing waves. For the left boundary this means $a_{1}=a_{3}=a_{4}=0$, as for the right boundary $a_{2}=0$ is required.

Let both the right-hand and the left-hand boundary condition be a linear constant-coefficient system of general form

$$
\left.\mathcal{L} U\right|_{x=x_{\Gamma}}=0,
$$


where operator $\mathcal{L}$ has the Fourier counterpart $\widehat{\mathcal{L}}(k, \ell, \omega)$. In [5] the amplitudes $a_{j}$ are shown to satisfy the system of linear algebraic equations

$$
\sum_{j=1}^{4} \widehat{V}_{j} a_{j}=0, \quad \text { where } \quad \widehat{V}_{j}=\widehat{\mathcal{L}}\left(k_{j}, \ell, \omega\right) \widehat{U}_{j}, \quad j=1,2,3,4 .
$$

In the case of 2D Euler equations there are no local nonreflecting boundary conditions. More precisely, acoustic waves of various incidence angles are inevitably reflected. In turn, entropy and vorticity waves are not reflected nor generated for a certain class of boundary conditions.

\section{FINITE-DIFFERENCE GASDYNAMIC SCHEMES}

Edge-Based Reconstruction (EBR) schemes [7] are designed for the nonlinear gas-dynamic equations on an arbitrary unstructured mesh and form a subcategory in finite-volume methods (FVM). A governing scheme is based on the conservation laws in flux representation and their subsequent discretization. Yet another feature of EBR is their vertex-centered structure. Generally, this kind of numerical schemes is comparatively easy in construction as well as in a posteriori analysis.

The further theory will be developed for the linearized Euler equations and a 2D rectangular spatial domain with a uniform mesh in each variable

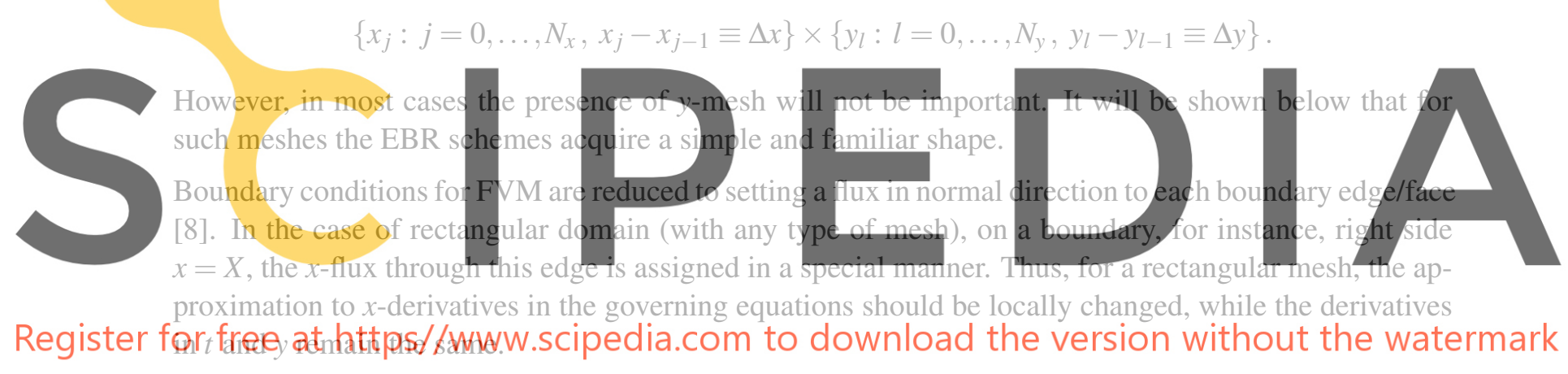

The advantage of such class of one-dimensional boundary conditions in multi-D is a simple and correct treatment of corner zones in a computational domain. Similar approaches to continuous boundary conditions can be found in $[9,10]$.

The disadvantage is the neglecting a possibility to improve boundary conditions by introducing appropriate tangential derivatives-see Ref. [4, 11, 12]. In this way one obtains local boundary conditions nonreflecting for vorticity waves in the Euler equations. Nevertheless, some opportunities exist in the case of discrete schemes.

As we previously mentioned in [3], a mechanism of wave propagation and reflection is distinct in continuous and discrete fluid models. In the linear case, various numerical schemes typically have two types of normal modes: regular — similar to those of the Euler equations_-and singular-additional scheme modes.

Centered-difference approximations to hyperbolic systems always possess grid-to-grid (sawtooth) oscillations which do not decay in space. Schemes with upwind differences have only exponentially dumping modes and such type of solutions are present in a number of centered-difference schemes. When an 


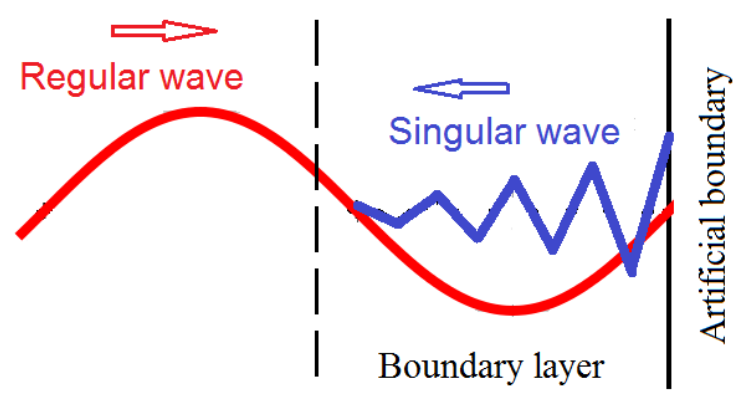

Figure 2: Schematic of the reflection of a regular wave in the form of singular wave

incident regular wave interacts with a boundary, regular and singular waves are generated with certain amplitudes (Fig. 2). In this case, the reflected fast-dumping scheme waves exist physically within a narrow area near the boundary (boundary layer).

All the numerical schemes to be considered here require a greater number of boundary conditions than the differential system. We will start with the linear 1D advection equation and then proceed to the Euler equations approximations.

3.1 Schemes for the advection equation

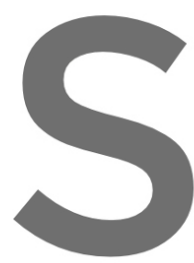

The Edge-Based Reconstruction

and then implemented to
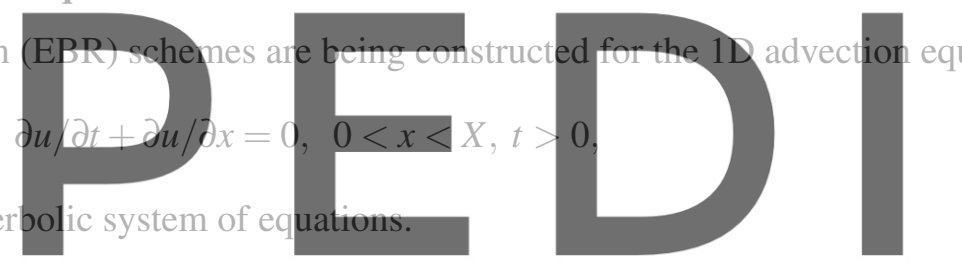

Eq. (10) is replaced, on a uniform mesh, with a discrete equation represented in the flux form as

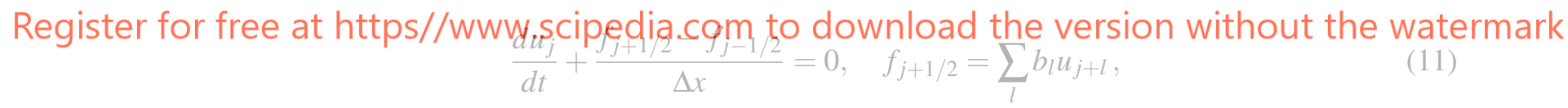

where $b_{l}$ form a finite set of known coefficients. The particular case of symmetric (centered-difference) schemes is characterized with relation

$$
b_{l}=b_{1-l} .
$$

In a numerical algorithm the time derivative $d / d t$ is implemented by using some Runge-Kutta method.

Eq. (11) has the general normal-mode solution

$$
u\left(x_{j}, t ; \omega\right)=a_{1} \exp \left\{i \omega t-i k_{1} x_{j}\right\}+\sum_{l} a_{l}^{S} \exp \left\{i \omega t-i k_{l}^{S} x_{j}\right\} .
$$

The regular mode is similar to the solution of continuous equation (10), and the corresponding wavenumber is

$$
k_{1}=\omega\left(1+O(\omega \Delta x)^{p}\right),
$$

where $p$ is the approximation order of scheme (11). 
The singular modes are expressed through wavenumbers

$$
k_{m}^{S}=\frac{i}{h} \ln q_{m}(1+O(\omega \Delta x))
$$

where $q=q_{m}$ are the roots of $f_{j+1 / 2}$ characteristic polynomial

$$
\sum_{l} b_{l} q^{l}=0
$$

In [3], examples of schemes were illustrated with exact analytic normal-mode solutions. However, for our further analysis, mostly sufficient will be the leading terms of expressions. This simplifies the procedure essentially.

Scheme (11) cannot be expressed in standard way at a number of near-boundary nodes. The corresponding fluxes $f$ should be altered. We consider this as the boundary conditions.

Upwind scheme EBR3:

$$
f_{j+1 / 2}=\frac{1}{6}\left(2 u_{j+1}+5 u_{j}-u_{j-1}\right), \quad \frac{d u_{j}}{d t}+\frac{2 u_{j+1}+3 u_{j}-6 u_{j-1}+u_{j-2}}{6 \Delta x}=0 .
$$

Polynomial (12) has the two roots $q_{1}$ and $q_{2}$, and the general normal-mode solution has the approximate (leading-term) form
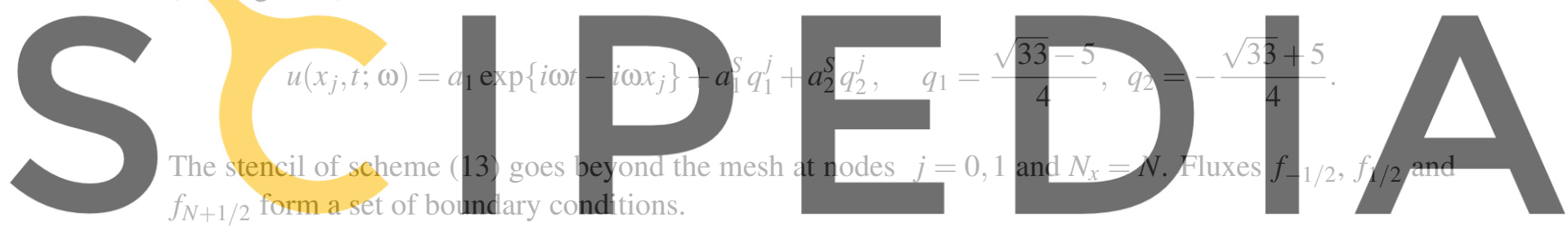

Quantity $f_{N+1 / 2}$ can be extrapolated from values of function $u$ within the domain. The linear extrapola-

Register forffrequat https//www.scipedia.com to download the version without the watermark

$$
f_{N+1 / 2}=\frac{1}{2}\left(3 u_{N}-u_{N-1}\right), \quad \frac{d u_{N}}{d t}+\frac{7 u_{N}-8 u_{N-1}+u_{N-2}}{6 \Delta x}=0 .
$$

The left-hand fluxes $f_{-1 / 2}$ and $f_{1 / 2}$ may be set equal to incoming values of $u=0$. Accordingly, the governing equation (13) is replaced at points 0 and 1 as follows:

$$
f_{-1 / 2}=f_{1 / 2}=0, \quad \frac{d u_{1}}{d t}+\frac{2 u_{2}+5 u_{1}-u_{0}}{6 \Delta x}=0, \quad \frac{d u_{0}}{d t}=0 .
$$

Symmetric scheme EBR4:

$$
f_{j+1 / 2}=\frac{1}{12}\left(-u_{j+2}+7 u_{j+1}+7 u_{j}-u_{j-1}\right), \quad \frac{d u_{j}}{d t}+\frac{u_{j-2}-8 u_{j-1}+8 u_{j+1}-u_{j+2}}{12 \Delta x}=0 .
$$

Polynomial (12) has the three roots

$$
q_{1}=-1, q_{2}=4+\sqrt{15}, q_{3}=4-\sqrt{15} .
$$


The first corresponds to the sawtooth mode and the others describe two fast-dumping exponents. More precisely, the spurious oscillation $k_{1}^{S}$ propagates leftward with speed acceleration of factor $5 / 3$. The general normal-mode solution to EBR4 scheme has the approximate form

$$
u\left(x_{j}, t ; \omega\right)=a_{1} \exp \left\{i \omega t-i \omega x_{j}\right\}+a_{1}^{S}(-1)^{j} \exp \left\{i \omega t+(5 / 3) i \omega x_{j}\right\}+a_{2}^{S} q_{2}^{j}+a_{3}^{S} q_{3}^{j} .
$$

The boundary conditions for EBR4 scheme are needed two on the left-hand edge and two on the righthand. As previously, set a flux extrapolation

$$
f_{N+1 / 2}=\frac{1}{2}\left(3 u_{N}-u_{N-1}\right), \quad f_{N-1 / 2}=\frac{1}{2}\left(3 u_{N-1}-u_{N-2}\right)
$$

and zero incoming fluxes

$$
f_{-1 / 2}=f_{1 / 2}=0
$$

The correspondent scheme coefficients can be obtained by combining (16) with (17) and (18).

\subsection{Schemes for the 2D Euler equations}

In upwind schemes for the linearized Euler equations, matrix $\mathbf{C}_{x}$ is split to "positive" and "negative" parts by means of factorization (7) as follows:

$$
\mathbf{C}_{x}=\mathbf{C}_{x}^{+}+\mathbf{C}_{x}^{-}, \quad \mathbf{C}_{x}^{+}=\mathbf{U} \Lambda_{x}^{+} \mathbf{U}^{-1}, \quad \Lambda_{x}^{+}=\operatorname{diag}\left\{\lambda_{j}^{+}\right\}=\operatorname{diag}(u+c, \quad 0, \quad u, u),
$$
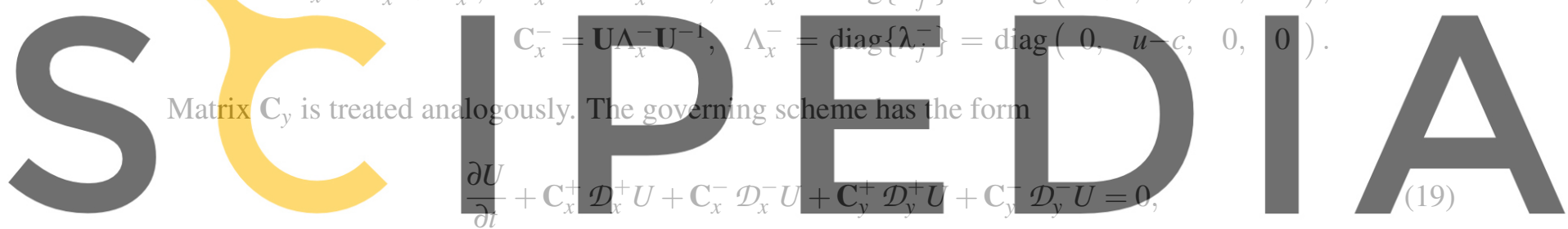

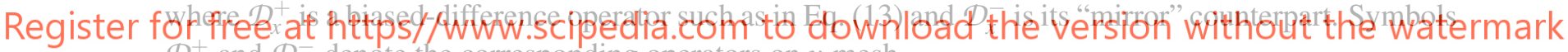 $\mathcal{D}_{y}^{+}$and $\mathcal{D}_{y}^{-}$denote the corresponding operators on $y$-mesh.}

Centered schemes can be expressed in form (19) as well. However, the symmetry of operators $\mathcal{D}_{x}^{+}=\mathcal{D}_{x}$ simplifies the governing numerical scheme to

$$
\frac{\partial U}{\partial t}+\mathbf{C}_{x} \mathcal{D}_{x}^{h} U+\mathbf{C}_{y} \mathcal{D}_{y}^{h} U=0
$$

Note that in the formulation of boundary conditions one should return to the general representation (19).

A solution to the finite-difference scheme is expanded over regular and singular modes

$$
U(x, y, t ; \omega, \ell)=\exp \{i \omega t-i \ell y\}\left[\sum_{j=1}^{4} a_{j} \widehat{U}_{j} \exp \left\{-i k_{j} x\right\}+\sum_{j} a_{j}^{S} \widehat{U}_{j}^{S} \exp \left\{-i k_{j}^{S} x\right\}\right]
$$

The regular modes $\left(\widehat{U}_{j}, k_{j}\right)$ are similar to the four physical modes of continuous Euler equations (5). The number and form of singular modes $\left(\widehat{U}_{j}^{S}, k_{j}^{S}\right)$ depend on the scheme considered. 
Boundary condition (9) specifies the following equation system for amplitudes

$$
\sum_{j=1}^{4} \widehat{V}_{j} a_{j}+\sum_{j} \widehat{V}_{j}^{S} a_{j}^{S}=0, \text { where } \widehat{V}_{j}=\widehat{\mathcal{L}}\left(k_{j}, \ell, \omega\right) \widehat{U}_{j}, \widehat{V}_{j}^{S}=\widehat{\mathcal{L}}\left(k_{j}^{S}, \ell, \omega\right) \widehat{U}_{j}^{S} .
$$

Nonreflecting boundary conditions for finite-difference schemes will be meant, following Ref. [3], in the simple (or "weak") sense. For arbitrary amplitudes of regular outgoing modes, the amplitudes of incoming regular modes do not depend on them. From the physical point of view, outgoing regular waves should not generate incoming regular waves. As stated in [3], this implies the structure of Eq. (21) solution:

$$
\begin{aligned}
& a_{j}=0 \text { for incoming modes, if } a_{l}^{S}=0, \forall l, \\
& a_{j} \text { arbitrary for outgoing modes. }
\end{aligned}
$$

For EBR3 scheme (13) the governing system of equations has the form (19):

$$
\frac{\partial U}{\partial t}+\mathbf{C}_{x}^{+} \mathcal{D}_{x}^{+} U+\mathbf{C}_{x}^{-} \mathcal{D}_{x}^{-} U+\mathbf{C}_{y}^{+} \mathcal{D}_{y}^{+} U+\mathbf{C}_{y}^{-} \mathcal{D}_{y}^{-} U=0 .
$$

Here operators $\mathcal{D}_{x}^{+}$and $\mathcal{D}_{x}^{-}$are

$$
\mathcal{D}_{x}^{+} u \equiv \frac{2 u_{j+1}+3 u_{j}-6 u_{j-1}+u_{j-2}}{6 \Delta x}, \mathcal{D}_{x}^{-} u \equiv \frac{-2 u_{j-1}-3 u_{j}+6 u_{j+1}-u_{j+2}}{6 \Delta x} .
$$

For scheme (22), the expansion over 12 normal modes looks in leading terms like
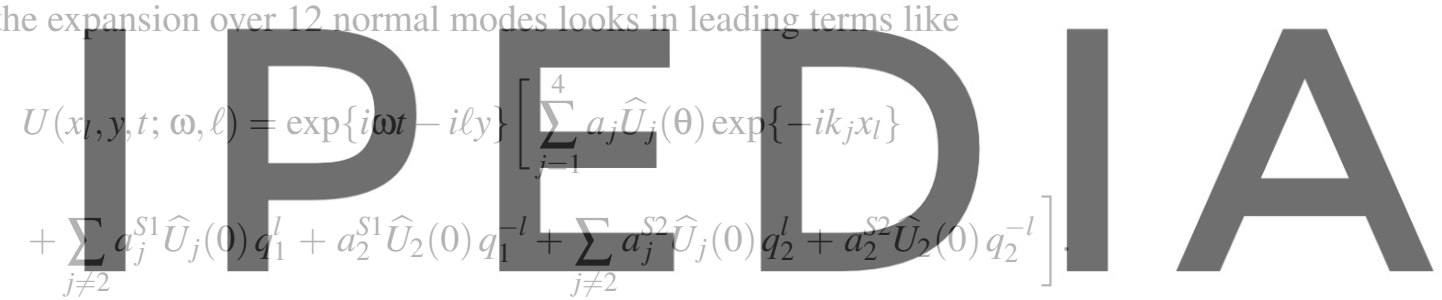

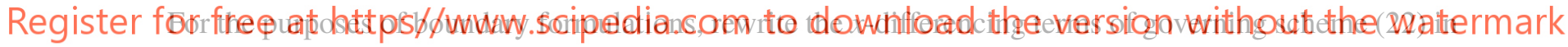
the flux form

$$
\left.\left(\mathbf{C}_{x}^{+} \mathcal{D}_{x}^{+} U+\mathbf{C}_{x}^{-} \mathcal{D}_{x}^{-} U\right)\right|_{x=x_{j}}=\left(F_{j+1 / 2}^{+}-F_{j-1 / 2}^{+}\right) \frac{1}{\Delta x}+\left(F_{j+1 / 2}^{-}-F_{j-1 / 2}^{-}\right) \frac{1}{\Delta x},
$$

where vectors $F_{j+1 / 2}^{+}$and $F_{j+1 / 2}^{-}$are, according to Eq. (13), constructed as

$$
\begin{array}{cl}
F_{j+1 / 2}^{+}=\mathbf{C}_{x}^{+} W_{j+1 / 2}^{+}, & F_{j+1 / 2}^{-}=\mathbf{C}_{x}^{-} W_{j+1 / 2}^{-}, \\
W_{j+1 / 2}^{+}=\frac{1}{6}\left(2 U_{j+1}+5 U_{j}-U_{j-1}\right), & W_{j+1 / 2}^{-}=\frac{1}{6}\left(2 U_{j}+5 U_{j+1}-U_{j+2}\right) .
\end{array}
$$

Now discuss the representation of EBR3 scheme at the boundary nodes. Like in Eqs. (14)-(15), variables $W^{+}$and $W^{-}$are altered in the following manner:

$$
\begin{gathered}
W_{N+1 / 2}^{+}=\frac{1}{2}\left(3 U_{N}-U_{N-1}\right), \quad W_{1 / 2}^{+}=0, \quad W_{-1 / 2}^{+}=0, \\
W_{-1 / 2}^{-}=\frac{1}{2}\left(3 U_{0}-U_{1}\right), \quad W_{N-1 / 2}^{-}=0, \quad W_{N+1 / 2}^{-}=0 .
\end{gathered}
$$


The task is to find vector relations $F^{+}\left(W^{+}\right)$and $F^{-}\left(W^{-}\right)$. The characteristic boundary conditions use the same expressions (23) for $j=-1,0, N-1, N$, i.e.,

$$
\begin{aligned}
& F_{-1 / 2}^{+}=\mathbf{C}_{x}^{+} W_{-1 / 2}^{+}, F_{-1 / 2}^{-}=\mathbf{C}_{x}^{-} W_{-1 / 2}^{-}, F_{1 / 2}^{-}=\mathbf{C}_{x}^{-} W_{1 / 2}^{-}, \\
& F_{N+1 / 2}^{+}=\mathbf{C}_{x}^{+} W_{N+1 / 2}^{+}, \quad F_{N+1 / 2}^{-}=\mathbf{C}_{x}^{-} W_{N+1 / 2}^{-}, F_{N-1 / 2}^{-}=\mathbf{C}_{x}^{-} W_{N-1 / 2}^{-} .
\end{aligned}
$$

There are alternative ways - the transformation matrix can be changed. A possible approach is based on radiation boundary conditions $[4,5]$. Here we change the technique, so far as the flux form of boundary conditions is used. As an example, impose the equations of left- and right-going acoustic waves on the corresponding sides of the domain:

$$
\begin{aligned}
& F_{-1 / 2}^{+}=F_{1 / 2}^{+}=0, \quad F_{-1 / 2}^{-}=(u-c) W_{-1 / 2}^{-}, \\
& F_{N+1 / 2}^{+}=(c+u) W_{N+1 / 2}^{+}, \quad F_{N+1 / 2}^{-}=F_{N-1 / 2}^{-}=0 .
\end{aligned}
$$

The centered scheme EBR4 from (16) produces the governing scheme (20) for the Euler equations

$$
\begin{aligned}
& \frac{\partial U}{\partial t}+\mathbf{C}_{x} \mathcal{D}_{x}^{h} U+\mathbf{C}_{y} \mathcal{D}_{y}^{h} U=0, \\
& \mathcal{D}_{x}^{h} u \equiv \frac{u_{j-2}-8 u_{j-1}+8 u_{j+1}-u_{j+2}}{12 \Delta x} .
\end{aligned}
$$

The solution to (25) has the following approximate expansion over 16 modes
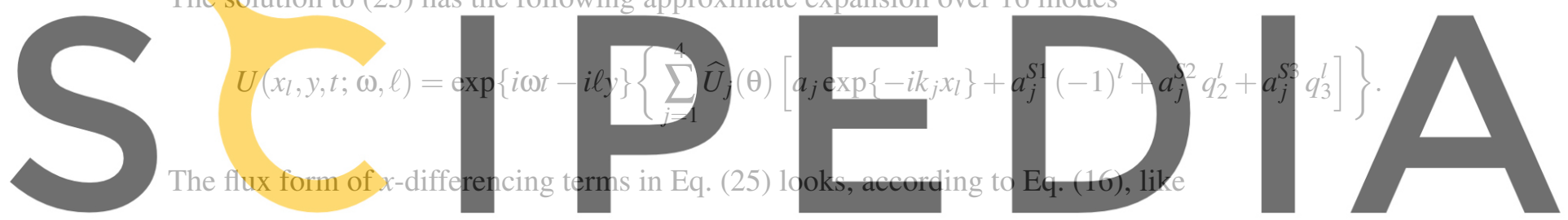

\section{$\mathbb{C}_{x} \mathcal{D}_{x}^{h} U\left(x_{j}, y, t\right)=\left(F_{j+1 / 2}-F_{j-1 / 2}\right) \frac{1}{A}, \quad F_{j+1 / 2}=\mathbf{C}_{x} \frac{1}{12}\left(-U_{j+2}+7 U_{j+1}+7 U_{j}-U_{j-1}\right)$.}

Register for free at https//www.scipedia.corn to download the version without the watermark

Near the boundaries a centered-difference scheme becomes similar to a scheme with biased differences.

EBR4 suggests spiitting the $x$-filuxes at nodes $j=-1 / 2,1 / 2, N-1 / 2$, and $N+1 / 2$ as

$$
F_{-1 / 2}=F_{-1 / 2}^{+}+F_{-1 / 2}^{-}, \quad F_{1 / 2}=F_{1 / 2}^{+}+F_{1 / 2}^{-}, \quad F_{N-1 / 2}=F_{N-1 / 2}^{+}+F_{N-1 / 2}^{-}, F_{N+1 / 2}=F_{N+1 / 2}^{+}+F_{N+1 / 2}^{-} .
$$

These fluxes are associated with the corresponding variables $W^{+}$and $W^{-}$whose form is taken from the scalar case (17)-(18):

$$
\begin{aligned}
& W_{N+1 / 2}^{+}=\frac{1}{2}\left(3 U_{N}-U_{N-1}\right), \quad W_{N-1 / 2}^{+}=\frac{1}{2}\left(3 U_{N-1}-U_{N-2}\right), \quad W_{1 / 2}^{+}=W_{-1 / 2}^{+}=0, \\
& W_{-1 / 2}^{-}=\frac{1}{2}\left(3 U_{0}-U_{1}\right), \quad W_{1 / 2}^{-}=\frac{1}{2}\left(3 U_{1}-U_{2}\right), \quad W_{N-1 / 2}^{-}=W_{N+1 / 2}^{-}=0 .
\end{aligned}
$$

The characteristic boundary conditions are:

$$
\begin{aligned}
& F_{-1 / 2}^{+}=\mathbf{C}_{x}^{+} W_{-1 / 2}^{+}, F_{-1 / 2}^{-}=\mathbf{C}_{x}^{-} W_{-1 / 2}^{-}, F_{1 / 2}^{+}=\mathbf{C}_{x}^{+} W_{1 / 2}^{+}, F_{1 / 2}^{-}=\mathbf{C}_{x}^{-} W_{1 / 2}^{-}, \\
& F_{N+1 / 2}^{+}=\mathbf{C}_{x}^{+} W_{N+1 / 2}^{+}, \quad F_{N+1 / 2}^{-}=\mathbf{C}_{x}^{-} W_{N+1 / 2}^{-}, \quad F_{N-1 / 2}^{+}=\mathbf{C}_{x}^{+} W_{N-1 / 2}^{+}, F_{N-1 / 2}^{-}=\mathbf{C}_{x}^{-} W_{N-1 / 2}^{-} .
\end{aligned}
$$


The acoustic radiation boundary conditions take the form:

$$
\begin{aligned}
& F_{-1 / 2}^{+}=F_{1 / 2}^{+}=0, \quad F_{-1 / 2}^{-}=(u-c) W_{-1 / 2}^{-}, \quad F_{1 / 2}^{-}=(u-c) W_{1 / 2}^{-}, \\
& F_{N+1 / 2}^{+}=(c+u) W_{N+1 / 2}^{+}, \quad F_{N-1 / 2}^{+}=(c+u) W_{N-1 / 2}^{+}, \quad F_{N+1 / 2}^{-}=F_{N-1 / 2}^{-}=0 .
\end{aligned}
$$

\section{CONCLUSION}

Finite-volume numerical schemes admit a variety of artificial boundary conditions. For EBR schemes with centered differences there exist boundary formulations which yield weak reflection of oblique waves. For biased-difference EBR schemes, boundary conditions are possible to diminish essentially the spurious reflection. The theoretical results in practice can be generalized to more complex models, including nonuniform or unstructured meshes and nonlinearity.

\section{REFERENCES}

[1] Engquist, B, and Majda, A. Absorbing boundary conditions for the numerical simulation of waves. Math. Comput. (1977) 31:629-651.

[2] Hu, F.Q. On absorbing boundary conditions for linearized Euler equations by a perfectly matched layer. J. Comput. Phys. (1996) 129:201-219.

[3] Abalakin, I.V. and Dorodnitsyn, L.V. Numerical simulation of subsonic gas flows using local discrete nonreflecting boundary conditions. ECCM 6 - ECFD 7. CIMNE, Barcelona (2018):740-750.

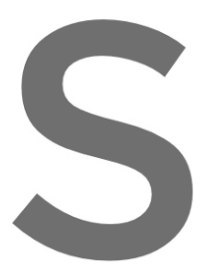

[4] Tam, C.K.W. and W
tational acoustics. J.
[5] Dorodnitsyn, L.V. A
Comput. Math. Math
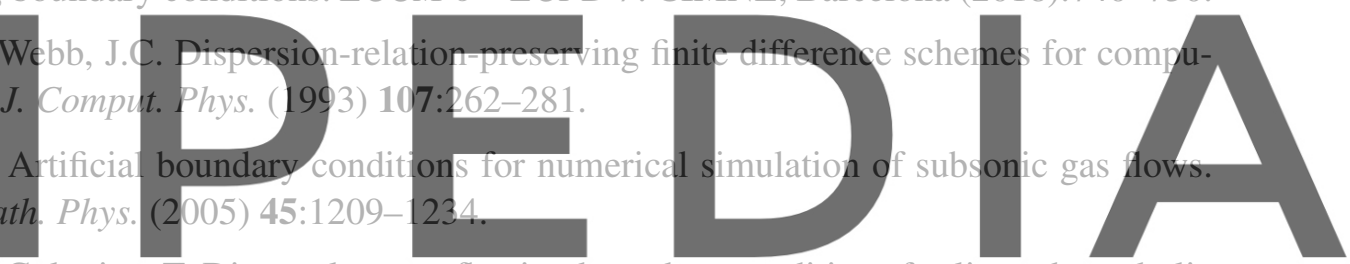

[6] Rowley, C.W. and Colonius, T. Discretely nonreflecting boundary conditions for linear hyperbolic Register for frystems, J.Comput. Phys. (2000) 157:500-538

[7] Abalakin, I., Bakhvalov, P., and Kozubskaya, T. Edge-based reconstruction schemes for unstructured tetrahedral meshes. Int. J. Numer. Meth. Fluids (2015) 81:331-356.

[8] Hirsch, C. Numerical computation of internal and external flows. Vol. 2: Computational methods for inviscid and viscous flows. Wiley, New York, (1990).

[9] Thompson, K.W. Time-dependent boundary conditions for hyperbolic systems. J. Comput. Phys. (1990) 89:439-461.

[10] Kroener, D. Absorbing boundary conditions for the linearized Euler equations in 2D. Math. Comp. (1991) 57:153-167.

[11] Giles, M.B. Nonreflecting boundary conditions for Euler equation calculations. AIAA J. (1990) 28:2050-2058.

[12] Dorodnicyn, L.W. Artificial boundary conditions for high-accuracy aeroacoustic algorithms. SIAM J. Scientific Computing (2010) 32:1950-1979. 